\title{
New physics and tau $g-2$ using LHC heavy ion collisions
}

\author{
Lydia Beresford $\odot^{1, *}$ and Jesse Liu $\circledast^{1,2, \dagger}$ \\ ${ }^{1}$ Department of Physics, University of Oxford, Oxford OX1 3RH, United Kingdom \\ ${ }^{2}$ Department of Physics, University of Chicago, Chicago, Illinois 60637, USA
}

(Received 1 November 2019; revised 5 April 2020; accepted 16 November 2020; published 22 December 2020)

\begin{abstract}
The anomalous magnetic moment of the tau lepton $a_{\tau}=\left(g_{\tau}-2\right) / 2$ strikingly evades measurement but is highly sensitive to new physics such as compositeness or supersymmetry. We propose using ultraperipheral heavy ion collisions at the LHC to probe modified magnetic $\delta a_{\tau}$ and electric dipole moments $\delta d_{\tau}$. We design a suite of analyses with signatures comprising one electron/muon plus track(s), leveraging the exceptionally clean photon fusion $\gamma \gamma \rightarrow \tau \tau$ events to reconstruct both leptonic and hadronic tau decays sensitive to $\delta a_{\tau}, \delta d_{\tau}$. Assuming $10 \%$ systematic uncertainties, the current $2 \mathrm{nb}^{-1}$ lead-lead dataset could already provide constraints of $-0.0080<a_{\tau}<0.0046$ at $68 \%$ C.L. This surpasses 15 -yearold lepton collider precision by a factor of 3 while opening novel avenues to new physics.
\end{abstract}

DOI: 10.1103/PhysRevD.102.113008

\section{INTRODUCTION}

Precision measurements of electromagnetic couplings are foundational tests of QED and powerful probes of beyond the Standard Model (BSM) physics. The electron anomalous magnetic moment $a_{e}=\frac{1}{2}\left(g_{e}-2\right)$ is among the most precisely known quantities in nature [1-5]. The muon counterpart $a_{\mu}$ is measured to $10^{-7}$ precision [6] and reports a $3-4 \sigma$ tension from Standard Model (SM) predictions [7-9]. This may indicate new physics [10-13], to be clarified at Fermilab [14] and J-PARC [15]. Measuring $a_{\ell}$ generically tests lepton compositeness [16], while supersymmetry at energy scales $M_{\mathrm{S}}$ induces radiative corrections $\delta a_{\ell} \sim m_{\ell}^{2} / M_{\mathrm{S}}^{2}$ for leptons with mass $m_{\ell}$ [10]. Thus, the tau $\tau$ can be $m_{\tau}^{2} / m_{\mu}^{2} \sim 280$ times more sensitive to BSM physics than $a_{\mu}$.

However, $a_{\tau}$ continues to evade measurement because the short tau proper lifetime of approximately $10^{-13}$ s precludes use of spin precession methods [6]. The most precise singleexperiment measurement $a_{\tau}^{\exp }$ is from DELPHI $[17,18]$ at the Large Electron Positron Collider (LEP) but is remarkably an order of magnitude away from the theoretical central value $a_{\tau, \mathrm{SM}}^{\mathrm{pred}}$ predicted to $10^{-5}$ precision [19]

$$
a_{\tau}^{\text {exp }}=-0.018(17), \quad a_{\tau, \mathrm{SM}}^{\mathrm{pred}}=0.00117721(5) .
$$

*lydia.beresford@physics.ox.ac.uk jesseliu@uchicago.edu

Published by the American Physical Society under the terms of the Creative Commons Attribution 4.0 International license. Further distribution of this work must maintain attribution to the author(s) and the published article's title, journal citation, and DOI. Funded by SCOAP ${ }^{3}$.
The poor constraints on $a_{\tau}$ present striking room for BSM physics, especially given other lepton sector tensions [20-32], and motivate new experimental strategies.

This paper proposes a suite of analyses to probe $a_{\tau}$ using heavy ion beams at the LHC. We leverage ultraperipheral collisions (UPCs) where only the electromagnetic fields surrounding lead $(\mathrm{Pb})$ ions interact. Tau pairs are produced from photon fusion $\mathrm{PbPb} \rightarrow$ $\mathrm{Pb}(\gamma \gamma \rightarrow \tau \tau) \mathrm{Pb}$, illustrated in Fig. 1, whose sensitivity to $a_{\tau}$ was suggested in 1991 [33]. We introduce the strategy crucial for experimental realization and importantly show that the currently recorded dataset could already surpass LEP precision. The LHC cross section enjoys a $Z^{4}$ enhancement $(Z=82$ for $\mathrm{Pb}$ ), with over 1 million $\gamma \gamma \rightarrow \tau \tau$ events produced to date. Existing proposals using lepton beams require future datasets (Belle-II) or proposed facilities (CLIC and LHeC) [34-41], while LHC studies focus on high luminosity proton beams [42-47]. No LHC analysis of

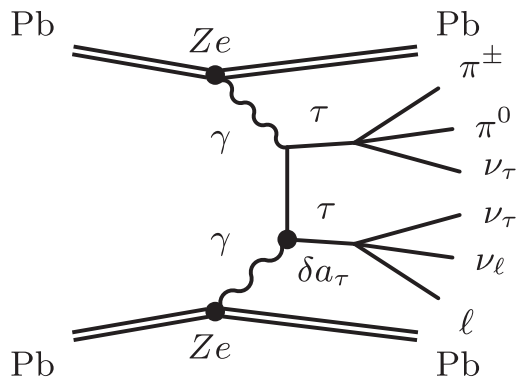

FIG. 1. Pair production of tau leptons $\tau$ from ultraperipheral lead ion $(\mathrm{Pb})$ collisions in two of the most common decay modes: $\pi^{ \pm} \pi^{0} \nu_{\tau}$ and $\ell \nu_{\ell} \nu_{\tau}$. New physics can modify tau-photon couplings affecting the magnetic moment by $\delta a_{\tau}$. 
$\gamma \gamma \rightarrow \tau \tau$ exists, as the taus have insufficient momentum for ATLAS/CMS to record or reconstruct.

Our proposal overcomes these obstructions in the clean UPC events [48], enabling selection of individual tracks from tau decays with no other detector activity akin to the LEP [17]. We exploit recent advances in low momentum electron/muon identification [49-51] to suppress hadronic backgrounds. We then present a shape analysis sensitive to interfering SM and BSM amplitudes to enhance $a_{\tau}$ constraints. Our strategy also probes tau electric dipole moments $d_{\tau}$ induced by $C P$ violating new physics. This opens key new directions in the heavy ion program amid reviving interest in photon collisions [52-54] for light-bylight scattering [55-58], standard candle processes [59-63], and BSM dynamics [64-74].

\section{EFFECTIVE THEORY AND PHOTON FLUX}

The anomalous $\tau$ magnetic moment $a_{\tau}=\left(g_{\tau}-2\right) / 2$ is defined by the spin-magnetic Hamiltonian $-\boldsymbol{\mu}_{\tau} \cdot \mathbf{B}=$ $-\left(g_{\tau} e / 2 m_{\tau}\right) \mathbf{S} \cdot \mathbf{B}$. In the Lagrangian formulation of QED, electromagnetic moments arise from the spinor tensor $\sigma^{\mu \nu}=\mathrm{i}\left[\gamma^{\mu}, \gamma^{\nu}\right] / 2$ structure of the fermion current interacting with the photon field strength $F_{\mu \nu}$,

$$
\mathcal{L}=\frac{1}{2} \bar{\tau}_{\mathrm{L}} \sigma^{\mu \nu}\left(a_{\tau} \frac{e}{2 m_{\tau}}-\mathrm{i} d_{\tau} \gamma_{5}\right) \tau_{\mathrm{R}} F_{\mu \nu} .
$$

Here, $\gamma^{5}$ satisfies the anticommutator $\left\{\gamma^{5}, \gamma^{\mu}\right\}=0$, and $\tau_{\mathrm{L}, \mathrm{R}}$ are tau spinors with L, R denoting chirality.

To introduce BSM modifications of $a_{\tau}$ and $d_{\tau}$, we use SM effective field theory (SMEFT) [75]. This assumes the scale of BSM physics $\Lambda$ is much higher than the probe momentum transfers $q$, i.e., $q^{2} \ll \Lambda^{2}$. At scale $q$, two dimension- 6 operators in the Warsaw basis [76] modify $a_{\tau}$ and $d_{\tau}$ at tree level, as discussed in Ref. [75],

$$
\mathcal{L}^{\prime}=\left(\bar{L}_{\tau} \sigma^{\mu \nu} \tau_{R}\right) H\left[\frac{C_{\tau B}}{\Lambda^{2}} B_{\mu \nu}+\frac{C_{\tau W}}{\Lambda^{2}} W_{\mu \nu}\right] .
$$

Here, $B_{\mu \nu}$ and $W_{\mu \nu}$ are the $\mathrm{U}(1)_{\mathrm{Y}}$ and $\mathrm{SU}(2)_{\mathrm{L}}$ field strengths, $H\left(L_{\tau}\right)$ is the Higgs (tau lepton) doublet, and $C_{i}$ are dimensionless, complex Wilson coefficients. We fix $C_{\tau W}=0$ to parametrize the two modified moments $\left(\delta a_{\tau}, \delta d_{\tau}\right)$ using two real parameters $\left(\left|C_{\tau B}\right| / \Lambda^{2}, \varphi\right)[40]$,

$$
\delta a_{\tau}=\frac{2 m_{\tau}}{e} \frac{\left|C_{\tau B}\right|}{M} \cos \varphi, \quad \delta d_{\tau}=\frac{\left|C_{\tau B}\right|}{M} \sin \varphi,
$$

where $\varphi$ is the complex phase of $C_{\tau B}$, we define $M=\Lambda^{2} /\left(\sqrt{2} v \cos \theta_{W}\right), \theta_{W}$ is the electroweak Weinberg angle, and $v=246 \mathrm{GeV}$.

In the SM, pair production of electrically charged particles $X$ from photon fusion $\gamma \gamma \rightarrow X X$ have analytic cross sections $\sigma_{\gamma \gamma \rightarrow X X}[71,77,78]$. For BSM variations, we employ the flavor-general SMEFTsim package [79], which implements Eq. (3) in FeynRules [80]. This allows a direct interface with MadGraph2.6.5 [81,82] for cross section calculation and Monte Carlo simulation. To model interference between SM and BSM diagrams, we generate $\gamma \gamma \rightarrow \tau \tau$ events with up to two BSM couplings $C_{\tau B}$ in the matrix element.

Turning to the source of photons, these are emitted coherently from electromagnetic fields surrounding the ultrarelativistic ions, which is known as the equivalent photon approximation [83]. We follow the MadGraph implementation in Ref. [84], which assumes the LHC exclusive cross section $\sigma_{\gamma \gamma \rightarrow X X}^{(\mathrm{PbP})}$ is factorized into a convolution of $\sigma_{\gamma \gamma \rightarrow X X}$ with the ion photon fluxes $n(x)$,

$$
\sigma_{\gamma \gamma \rightarrow X X}^{(\mathrm{PbPb})}=\int \mathrm{d} x_{1} \mathrm{~d} x_{2} n\left(x_{1}\right) n\left(x_{2}\right) \sigma_{\gamma \gamma \rightarrow X X},
$$

where $x_{i}=E_{i} / E_{\text {beam }}$ is the ratio of the emitted photon energy $E_{i}$ from ion $i$ with beam energy $E_{\text {beam. }}$. In this factorized prescription, $n(x)$ assumes an analytic form from classical field theory $[84,85]$,

$n(x)=\frac{2 Z^{2} \alpha}{x \pi}\left\{\bar{x} K_{0}(\bar{x}) K_{1}(\bar{x})-\frac{\bar{x}^{2}}{2}\left[K_{1}^{2}(\bar{x})-K_{0}^{2}(\bar{x})\right]\right\}$,

where $\bar{x}=x m_{N} b_{\min }, m_{N}$ is the nucleon mass $m_{N}=$ $0.9315 \mathrm{GeV}$, and $Z=82$ for $\mathrm{Pb}$. We set the minimum impact parameter $b_{\min }$ to be the nuclear radius $b_{\min }=R_{A} \simeq$ $1.2 A^{1 / 3} \mathrm{fm}=6.09 A^{1 / 3} \mathrm{GeV}^{-1}$, where $A=208$ is the mass number of $\mathrm{Pb}$ used at the LHC. We use Ref. [86] to numerically evaluate the modified Bessel functions of the second kind of first $K_{0}$ and second $K_{1}$ order.

We modify MadGraph to use the photon flux Eq. (6) for evaluating $\sigma_{\gamma \gamma \rightarrow X X}^{(\mathrm{PbPb})}$. This prescription neglects a nonfactorizable term in Eq. (5), which models the probability of hadronic interactions $P_{\left|\mathbf{b}_{1}-\mathbf{b}_{2}\right|}$, where $\mathbf{b}_{i}$ is the impact parameter of ion $i$. The SuperChic3.02 [87] program includes a complete treatment of $P_{\left|\mathbf{b}_{1}-\mathbf{b}_{2}\right|}$, along with nuclear overlap and thickness. Using this, we validate that these simplifications in MadGraph do not majorly impact distributions relevant for this work, namely tau $p_{\mathrm{T}}$. We generate 3 million $\gamma \gamma \rightarrow \tau \tau$ events for each coupling variation at $\sqrt{s_{\mathrm{NN}}}=5.02 \mathrm{TeV}$. For the $\mathrm{SM}$, we find $\sigma_{\gamma \gamma \rightarrow \tau \tau}^{(\mathrm{PbPb})}=$ $5.7 \times 10^{5} \mathrm{nb}$. To improve generator statistics, we impose $p_{\mathrm{T}}^{\tau}>3 \mathrm{GeV}$ in MadGraph, which has a $21 \%$ efficiency. Due to destructive interference, $\sigma_{\gamma \gamma \rightarrow \tau \tau}^{(\mathrm{PbP})}$ falls to a minimum of $4.7 \times 10^{5} \mathrm{nb}$ at $\delta a_{\tau} \simeq-0.04$ before returning to $5.7 \times 10^{5} \mathrm{nb}$ at $\delta a_{\tau} \simeq-0.09$. Further validation of these effects is in the Appendix A. We employ PYTHIA8.230 [88] for decay, shower, and hadronization, then use DELPHES3.4.1 [89] for detector emulation. 


\section{PROPOSED ANALYSES}

To record $\gamma \gamma \rightarrow \tau \tau$ events, dedicated UPC triggers are crucial for our proposal. With no other detector activity, the ditau system receives negligible transverse boost, and each tau $p_{\mathrm{T}}$ reaches a few to tens of $\mathrm{GeV}$ at most. Taus always decay to a neutrino $\nu_{\tau}$, which further dilutes the visible momenta, rendering usual hadronic tau triggers $p_{\mathrm{T}}(\tau$-jet $) \gtrsim 20 \mathrm{GeV}$ unfeasible [90,91]. However, UPC events without pileup enable exceptionally low trigger thresholds by vetoing large sums over calorimeter transverse energy deposits $\sum E_{\mathrm{T}}<50 \mathrm{GeV}$ [58]. Other minimum bias triggers are also possible [92,93]. A recent UPC dimuon analysis additionally requires at least one track and no explicit $p_{\mathrm{T}}$ requirement for the trigger muon [63]. The light-by-light observation also considers ultralow $E_{\mathrm{T}}>$ $1 \mathrm{GeV}$ calorimeter cluster thresholds at trigger level [58], which can similarly benefit electrons.

We design our event selection around two objectives. First, we consider standard objects already deployed by ATLAS/CMS to efficiently reconstruct tau decays with the following branching fractions [18]:

$$
\begin{gathered}
\mathcal{B}\left(\tau^{ \pm} \rightarrow \ell^{ \pm} \nu_{\ell} \nu_{\tau}\right)=35 \%, \\
\mathcal{B}\left(\tau^{ \pm} \rightarrow \pi^{ \pm} \nu_{\tau}+\text { neutral pions }\right)=45.6 \%, \\
\mathcal{B}\left(\tau^{ \pm} \rightarrow \pi^{ \pm} \pi^{\mp} \pi^{ \pm} \nu_{\tau}+\text { neutral pions }\right)=19.4 \% .
\end{gathered}
$$

We develop signal regions (SRs) targeting these decays based on expected signal rate and background mitigation strategies. We impose the lowest trigger and reconstruction thresholds $p_{\mathrm{T}}^{e / \mu}>4.5 / 3 \mathrm{GeV},\left|\eta_{e / \mu}\right|<2.5 / 2.4$ supported by ATLAS/CMS $[49,50]$. Second, we optimize sensitivity to different couplings $\delta a_{\tau}, \delta d_{\tau}$, where interfering SM and BSM amplitudes impact tau kinematics, which propagates to, e.g., lepton $p_{\mathrm{T}}$. The following analyses are considered:

Dilepton analysis. Requiring two identified leptons is expected to give the highest signal-to-background $S / B$, with half being different flavor $e \mu$ free of $e e / \mu \mu$ backgrounds. But even using low $p_{\mathrm{T}}^{e / \mu}$ thresholds, we find insufficient signal yields at $2 \mathrm{nb}^{-1}$ to pursue this further.

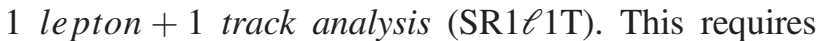
exactly one identified lepton and one other track that is not "matched" to the lepton [the matched track is the highest $p_{\mathrm{T}}$ track with $\Delta R(\ell$, track $\left.)<0.02\right]$. Tracks must satisfy the standard requirements $p_{\mathrm{T}}^{\text {track }}>$ $500 \mathrm{MeV}$ and $\left|\eta^{\text {track }}\right|<2.5$. With the DELPHES simulation, the requirement that the nonlepton track is not identified as a lepton allows for a sufficiently strong suppression of the dielectron/dimuon background, but for the analysis of experimental data, additional lepton veto selections may be considered to increase the background rejection at a modest cost to signal efficiency. This topology targets the high branching ratio of the single charged pion decay mode and background suppression from lepton identification. The track also recovers events failing the dilepton analysis, in which a lepton is too soft to be reconstructed. We divide this SR into two bins $p_{\mathrm{T}}^{e / \mu} \in$ $[\leq 6],[>6] \mathrm{GeV}$ to exploit shape differences shown in Fig. 2 (left). We require nonplanar lepton-track system $\mid \Delta \phi(\ell$, trk $) \mid<3$ to further suppress $e e / \mu \mu$ processes, as demonstrated in Fig. 2 (right). We veto invariant masses $m_{\ell, \text { trk }} \notin[3,3.2],[9,11] \mathrm{GeV}$ to reject dilepton decays of $J / \psi$ and $\Upsilon$ resonances.

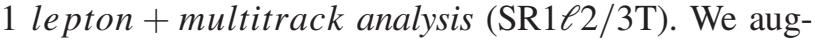
ment the previous analysis with three non-leptonmatched tracks. This targets the decay topology with three charged pions. We also construct an orthogonal SR requiring exactly two tracks to recover misreconstructed three-pion decays. By taking the vectorial sum over $p_{\mathrm{T}}$ for the non-lepton-matched tracks $p_{\mathrm{T}}^{\text {tracks }}=\sum_{i} p_{\mathrm{T}, i}^{\mathrm{track}}$, we obtain the track system $p_{\mathrm{T}}$, whose distribution is shown in Fig. 2 (center) for SR1 $\ell 3 \mathrm{~T}$. This variable shows discrimination power between modified moments and could be considered in future work.

Motivated by the background processes observed by DELPHI in $\gamma \gamma \rightarrow \tau \tau$ analyses, we now outline our
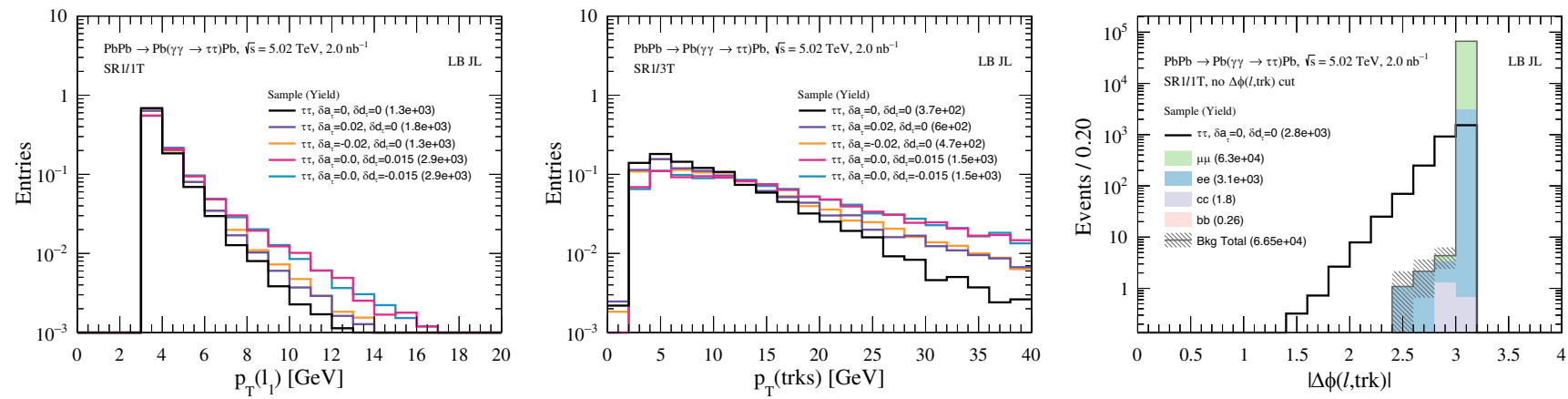

FIG. 2. Distributions of lepton $p_{\mathrm{T}}$ in SR1 $\ell 1 \mathrm{~T}$ (left) and the three-track system $p_{\mathrm{T}}$ in $\mathrm{SR} 1 \ell 3 \mathrm{~T}$ (center) for benchmark signals with various $\delta a_{\tau}, \delta d_{\tau}$ couplings. These are normalized to unit integral to illustrate shape changes with varying $\delta a_{\tau}, \delta d_{\tau}$. The lepton-track azimuthal angle $\mid \Delta \phi(\ell$, trk $) \mid$ in SR1 $\ell 1 \mathrm{~T}$ (right) is shown for backgrounds (filled) and signal $\delta a_{\tau}=\delta d_{\tau}=0$ (line), illustrating powerful discrimination against dilepton processes. 
considered backgrounds and mitigation strategies. Leptonic backgrounds are dominated by dielectron/dimuon production $\gamma \gamma \rightarrow \ell \ell, \ell \in[e, \mu]$. The single flavor cross section is sizable $\sigma_{\gamma \gamma \rightarrow \ell \ell}^{(\mathrm{PbPb})}=4.2 \times 10^{5} \mathrm{nb}$, which includes a generator level $\left|\eta_{\ell}\right|<2.5$ requirement. The back-to-back leptons are suppressed by requiring exactly 1 identified lepton in the event and $\mid \Delta \phi(\ell$, trk $) \mid<3$, which we verify by generating 1 million events per flavor. Photon radiation from leptons $\ell \rightarrow \ell \gamma$ is only expected to modify the tails marginally. Track impact parameters exploiting displaced tau decays could further suppress this background.

Hadronic backgrounds arise from diquark production $\gamma \gamma \rightarrow$ $q \bar{q}$, and we generate 1 million events for each of the five flavors. For $q \in[u, d, s]$, assuming massless quarks gives a cross section $\sigma_{\gamma \gamma \rightarrow u \bar{u}(d \bar{d}, s \bar{s})}^{(\mathrm{PbPb})}=3.0 \times 10^{5} \quad\left(1.9 \times 10^{4}\right) \mathrm{nb}$. Parton showering produces more tracks than tau decays, which we suppress using lepton isolation and requiring no more than four tracks at most. For $q \in[c, b]$, heavy flavor $B$ and $D$ mesons undergo semileptonic decays, e.g., $D \rightarrow \pi^{0} \ell \nu$. The default MadGraph parameters assume massless charm quarks (which is conservative as a finite mass decreases cross sections), yielding $\sigma_{\gamma \gamma \rightarrow c \bar{c}}^{(\mathrm{PbPb})}=3.0 \times 10^{5} \mathrm{nb}$. Bottom quarks assume finite mass, resulting in a smaller cross section $\sigma_{\gamma \gamma \rightarrow b \bar{b}}^{(\mathrm{PbPb})}=1.5 \times 10^{3} \mathrm{nb}$. The leptonic branching fraction $D \rightarrow \pi^{0} \ell \nu$ is of order a few percent and so is under control and is further suppressed by isolation.

Smaller potential backgrounds include $\gamma \gamma \rightarrow W W$, but the cross section $\sigma_{\gamma \gamma \rightarrow W W}^{(\mathrm{PbPb})}=14 \mathrm{pb}$ implies this is safely neglected. Exchange of digluon color singlets (Pomerons) also contributes to diquark backgrounds. These involve strong interactions, and as the binding energy per nucleon is very small at approximately $8 \mathrm{MeV}$ [84], the $\mathrm{Pb}$ ions emit more neutrons than $\mathrm{QED}$ processes, which can be vetoed by the Zero Degree Calorimeter (ZDC) [94]. Soft survival for Pomeron exchange is also lower [84], which gives greater activity in the calorimeter and tracker, and is suppressed by our stringent exclusivity requirements. We have crosschecked our simulation with the dimuon rate observed by a recent CMS analysis [95] when events with one or more neutrons recorded by the ZDC are vetoed, demonstrating that nucleon dissociation effects are sufficiently under control.

Systematic uncertainties require LHC collaborations to reliably quantify, but we discuss expected sources and suggest control strategies. Experimental systematics from current UPC $\mathrm{PbPb}$ dimuon measurements have systematics of around 10\%, dominated by luminosity and trigger [63]. Systematics from lepton reconstruction are $p_{\mathrm{T}}^{\ell}$ dependent and thus sensitive to $\delta a_{\tau}$. These are most significant at low $p_{\mathrm{T}}$ but are currently determined in high luminosity proton collisions with challenging backgrounds from fakes $[96,97]$ and could be better controlled using clean $\gamma \gamma \rightarrow \ell \ell$ events.

Theoretical uncertainties are expected to be dominated by modeling of the photon flux, nuclear form factors, and nucleon dissociation. Fortunately, these initial state effects are independent of the QED process and final state. So, experimentalists could use a control sample of $\gamma \gamma \rightarrow \ell \ell$ events to constrain these universal nuclear systematics or eliminate them in a ratio analysis with dileptons $\sigma_{\gamma \gamma \rightarrow \tau \tau}^{(\mathrm{PbPb})} / \sigma_{\gamma \gamma \rightarrow \ell \ell}^{(\mathrm{PbPb})}$. Hadronic backgrounds are susceptible to uncertainties from modeling the parton shower but are subdominant given $S / B \gg 1$ in our analyses.

\section{RESULTS AND DISCUSSION}

We now estimate the sensitivity of our analyses to modified tau moments $\delta a_{\tau}, \delta d_{\tau}$. Assuming the observed data correspond to the SM expectation, we calculate

$$
\chi^{2}=\frac{\left(S_{\mathrm{SM}+\mathrm{BSM}}-S_{\mathrm{SM}}\right)^{2}}{B+S_{\mathrm{SM}+\mathrm{BSM}}+\left(\zeta_{s} S_{\mathrm{SM}+\mathrm{BSM}}\right)^{2}+\left(\zeta_{b} B\right)^{2}} .
$$

Here, $B$ is the background rate, and $S_{\mathrm{SM}}\left(S_{\mathrm{SM}+\mathrm{BSM}}\right)$ is the expected $\gamma \gamma \rightarrow \tau \tau$ yield assuming SM couplings (nonzero $\left.\delta a_{\tau}, \delta d_{\tau}\right)$. At $\mathcal{L}=2 \mathrm{nb}^{-1}$, we find $S_{\mathrm{SM}}=1275.1, B=7.6$ for SR $1 \ell 1 \mathrm{~T}$ before binning in $p_{\mathrm{T}}^{\ell} ; S_{\mathrm{SM}}=519.9, B=15.4$ for SR $1 \ell 2 \mathrm{~T}$; and $S_{\mathrm{SM}}=370.5, B=4.0$ for SR $1 \ell 3 \mathrm{~T}$. We denote the relative signal (background) systematic uncertainties by $\zeta_{s}\left(\zeta_{b}\right)$ and study $\zeta_{s}=\zeta_{b} \in[5 \%, 10 \%]$ as benchmarks. For simplicity, we assume identical $\zeta_{s}$ for all couplings and combine the four SRs (SR1 $\ell 1 \mathrm{~T}$ has two $p_{\mathrm{T}}^{\ell}$ bins) using $\chi^{2}=\sum \chi_{\mathrm{SR}}^{2}$ assuming uncorrelated systematics. Note that, due to the high $S / B$ for each of the SRs, the systematic uncertainty on the background estimate has limited impact. We define the $68 \%$ C.L. (95\% C.L.) regions as couplings satisfying $\chi^{2}<1\left(\chi^{2}<3.84\right)$. Appendix B details cutflows for signals and backgrounds and $\chi^{2}$ distributions.

Figure 3 summarizes our projected $a_{\tau}=a_{\tau, \mathrm{SM}}^{\mathrm{pred}}+\delta a_{\tau}$ constraints (green) compared with existing measurements and predictions. Assuming the current dataset $\mathcal{L}=2 \mathrm{nb}^{-1}$ with $10 \%$ systematics, we find $-0.0080<a_{\tau}<0.0046$ at $68 \%$ C.L., surpassing DELPHI precision [17] (blue) by a factor of 3 . Negative values of $\delta a_{\tau}$ are more difficult to constrain, given destructive interference. We estimate prospects assuming halved systematics giving $-0.0022<$ $a_{\tau}<0.0037$ (68\% C.L.). A tenfold dataset increase for the High Luminosity LHC reduces this to $-0.00044<a_{\tau}<$ 0.0032 (68\% C.L.), an order of magnitude improvement beyond DELPHI. Importantly, these advances start constraining the sign of $a_{\tau}$ and become comparable to the predicted SM central value for the first time.

Such precision indirectly probes BSM physics. In nature, compositeness can induce large and negative magnetic moments, e.g., the neutron [18]. As a benchmark, we fix $C_{\tau B}=-1, C_{\tau W}=0, \delta d_{\tau}=0$ in Eq. (3) to recast the DELPHI limit into a $95 \%$ C.L. exclusion of $\Lambda<140 \mathrm{GeV}$. The orange line in Fig. 3 shows $140<\Lambda<250 \mathrm{GeV}$, where our $2 \mathrm{nb}^{-1}, 10 \%$ systematics proposal has $95 \%$ C.L. 


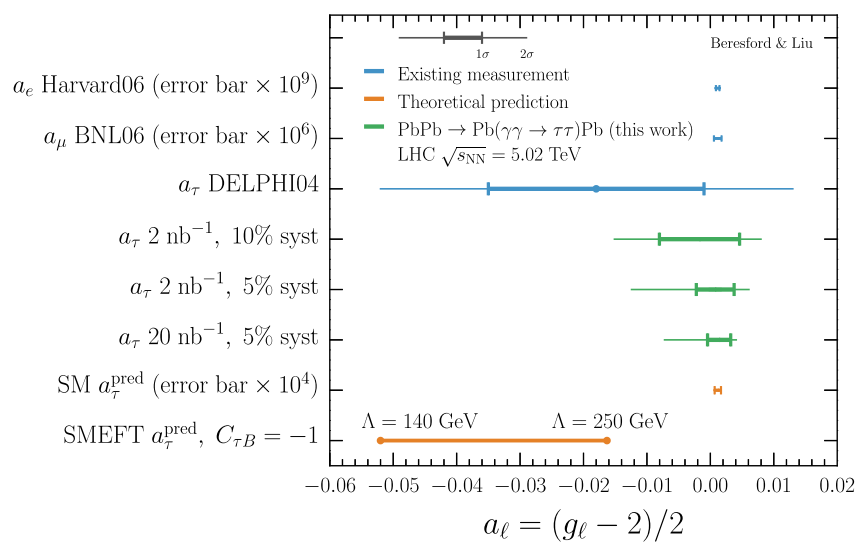

FIG. 3. Summary of lepton anomalous magnetic moments $a_{\ell}=\left(g_{\ell}-2\right) / 2$. Existing single-experiment measurements of $a_{e}$ [1], $a_{\mu}$ [6], and $a_{\tau}$ [17] are in blue. Our benchmark projections (green) assume $2 \mathrm{nb}^{-1}$ and $20 \mathrm{nb}^{-1}$ for $5 \%$ and $10 \%$ systematic uncertainties. For visual clarity, we inflate $1 \sigma$ error bars on $a_{e}\left(a_{\mu}\right)$ measurements by $10^{9}\left(10^{6}\right)$ and $10^{4}$ for the SM prediction $a_{\tau}^{\text {pred }}$ (orange) [19]. Collider constraints have thick (thin) lines denoting $68 \%$ C.L., $1 \sigma$ (95\% C.L, $\sim 2 \sigma)$. The SMEFT predictions $[75,76]$ from Eq. (4) with $C_{\tau B}=-1$ displays BSM scales $140<\Lambda<$ $250 \mathrm{GeV}$ (thick orange).

sensitivity, surpassing DELPHI by $110 \mathrm{GeV}$. In suitable ultraviolet completions of SMEFT with composite leptons, one can interpret $\Lambda$ as the confinement scale of tau substructure [16]. Nonetheless, our analyses are highly model independent, and we defer sensitivity to other BSM scenarios for future work. It would be interesting to correlate $a_{\tau}$ with models that simultaneously explain tensions in $a_{e}$ and $a_{\mu}$ [20-23] or $B$-physics lepton universality tests [24-28].

Lepton electric dipole moments are highly suppressed in the SM, arising only at four-loop $\left|d_{\tau}^{\text {pred }}\right| \sim\left(m_{\tau} / m_{e}\right)\left|d_{e}^{\text {pred }}\right| \sim$ $10^{-33} \mathrm{e} \mathrm{cm} \mathrm{[98].} \mathrm{Additional} C P$ violation in the lepton sector can enhance this, such as neutrino mixing [99] or other BSM physics parametrized by $\varphi$ in Eq. (4). Our projected $95 \%$ C.L. sensitivity on $d_{\tau}=\left(e / m_{\tau}\right) \delta d_{\tau}$ is $\left|d_{\tau}\right|<3.4 \times 10^{-17} e \mathrm{~cm}$, assuming $\delta a_{\tau}=0$ with $2 \mathrm{nb}^{-1}$, $10 \%$ systematics. This is an order of magnitude better than DELPHI $\left|d_{\tau}\right|<3.7 \times 10^{-16} e \mathrm{~cm}$ [17] and competitive with Belle [100].

Our proposal opens numerous avenues for extension. Lowering lepton/track thresholds to increase statistics would enable more optimized differential or multivariate analyses. Recently, ATLAS considered tracks matched to lepton candidates failing quality requirements, allowing $p_{\mathrm{T}}^{\text {track }}(e / \mu)>1 / 2 \mathrm{GeV}$ [51]. Moreover, the $500 \mathrm{MeV}$ track threshold is conservative, given $p_{\mathrm{T}}^{\text {track }}>100 \mathrm{MeV}$ is successfully used in ATLAS [58]. Reconstructing soft calorimeter clusters could enable hadron/electron identification, or using neutral pions to improve tau momentum resolution. Proposed timing detectors may offer more robust particle identification in ATLAS/CMS [101], while ALICE already has such capabilities [102]. Ultimate $a_{\tau}$ precision requires a coordinated worldwide program led by LHC efforts combined with proton-lead collisions at $\sqrt{s_{\mathrm{NN}}}=8.76 \mathrm{TeV}$, the Relativistic Heavy Ion Collider, and lepton colliders.

To summarize, we proposed a strategy of lepton plus track(s) analyses to surpass LEP constraints on tau electromagnetic moments using heavy ion data already recorded by the LHC. The clean photon collision events provide excellent opportunities to optimize low momentum reconstruction and control systematics further. We encourage LHC collaborations to open these cornerstone measurements and precision pathways to new physics.

\section{ACKNOWLEDGMENTS}

We are thankful for the hospitality of the LHC Forward and Diffractive Physics Workshop at CERN, where part of this work began. We are grateful to Luca Ambroz, Bill Balunas, Alan Barr, Mikkel Bjørn, Barak Gruberg, Lucian Harland-Lang, Simon Knapen, Santiago Paredes, Hannah Pullen, Hayden Smith, Beojan Stanislaus, Gabija Žemaityte, and Miha Zgubič for helpful discussions. L. B. is supported by a Junior Research Fellowship at St. John's College, Oxford. J. L. is supported by a STFC Postgraduate Studentship at Oxford, where this work started, and the Grainger Fellowship.

\section{APPENDIX A: SIMULATION VALIDATION}

We present additional material to validate the technical implementation of our simulation setup within the scope of our work. This includes the photon flux we implemented in MadGraph2.6.5 [81,82] and the interface with SMEFTsim [79] for BSM modifications and interference with the SM.

Figure 4 displays generator level differential distributions of $p_{\mathrm{T}}(\tau)$ for $\gamma \gamma \rightarrow \tau \tau$ considering various photon fluxes from protons and lead $(\mathrm{Pb})$ beams. The distribution generated in MadGraph with $\mathrm{Pb}$ uses our custom implementation of $\mathrm{Pb}$ ion photon flux. We validate this with the corresponding distribution generated in SuperChic 3.02 [87]. The latter includes a full treatment of nuclear effects that are neglected by the factorized prescription in MadGraph. These two distributions are in reasonable agreement for the scope of our work. Also shown are the corresponding distributions for proton beams. This illustrates that the impact of a nucleus with comparatively finite size is to soften the $p_{\mathrm{T}}(\tau)$ spectrum compared to using proton beams.

Figure 5 shows the impact of the interference behavior on the inclusive cross sections of $\sigma_{\gamma \gamma \rightarrow \tau \tau}^{(P b P b)}$ for coupling variations $\delta a_{\tau}$ using SMEFTsim. We account for the 

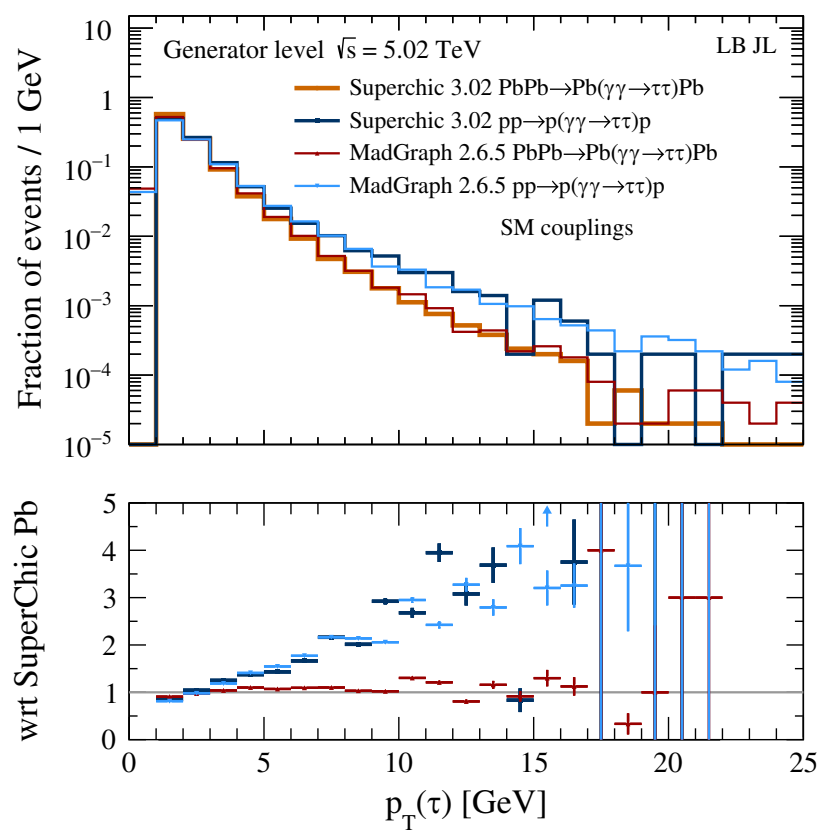

FIG. 4. Unit normalized generator level tau $p_{\mathrm{T}}$ distributions for $\gamma \gamma \rightarrow \tau \tau$ using SM couplings. These are generated in superchic3.02, which includes a full treatment of nuclear effects for lead $(\mathrm{Pb})$ ions (orange). Also shown is the corresponding sample with protons (dark blue). The MadGraph2.6.5 samples use a factorized photon flux prescription for protons (light blue) and our implementation of $\mathrm{Pb}$ ion flux (red). The ratio panel is with respect to the superchic $\mathrm{Pb}$ ions sample.

interference between SM and BSM $\gamma \gamma \rightarrow \tau \tau$ diagrams in the matrix element $\mathcal{M}$ squared,

$$
|\mathcal{M}|^{2}=\left|\mathcal{M}_{\mathrm{SM}}+\mathcal{M}_{\mathrm{BSM}}^{(1)}+\mathcal{M}_{\mathrm{BSM}}^{(2)}\right|^{2}
$$

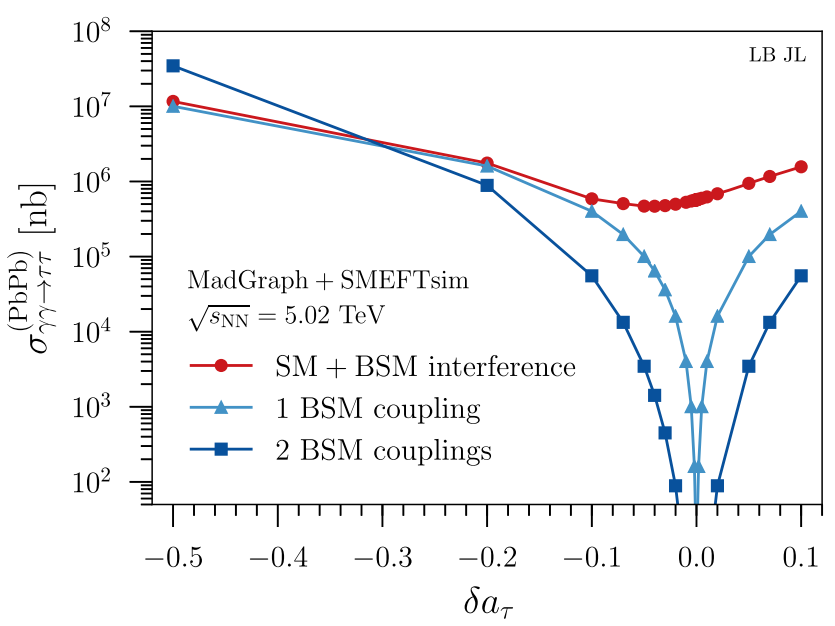

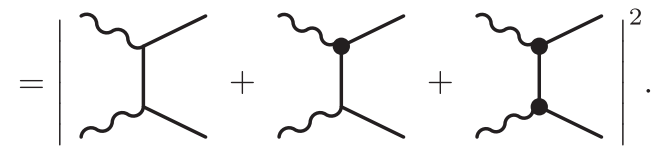

A BSM coupling is represented by (filled circle) in the matrix element diagrams. Cross sections featuring just the diagrams with only one BSM coupling (blue triangle) and only two BSM couplings (blue square) are shown in Fig. 5 and correspond to the amplitudes $\mathcal{M}_{\mathrm{BSM}}^{(1)}$ and $\mathcal{M}_{\mathrm{BSM}}^{(2)}$, respectively. As $\delta a_{\tau}$ deviates from zero in the negative direction, $\sigma_{\gamma \gamma \rightarrow \tau \tau}^{(\mathrm{PbPb})}$ falls to a minimum at $\delta a_{\tau} \simeq-0.04$ due to destructive interference from $\mathcal{M}_{\mathrm{BSM}}^{(1)}$. Then, the constructively interfering $\mathcal{M}_{\mathrm{BSM}}^{(2)}$ term begins to dominate for more negative $\delta a_{\tau}$ values, and $\sigma_{\gamma \gamma \rightarrow \tau \tau}^{(\mathrm{PbP})}$ rises again.

\section{APPENDIX B: CUTFLOWS AND $\chi^{2}$ DISTRIBUTIONS}

We provide technical material supporting the results presented in the main text. These include signal and background counts after sequentially applying kinematic requirements (cutflow) and $\chi^{2}$ distributions as functions of $\delta a_{\tau}$ and $\delta d_{\tau}$ used to derive the final constraints.

Table I presents the set of cutflows for the different analyses, sequentially displaying the yields normalized to $2 \mathrm{nb}^{-1}$ after each signal region requirement. Three benchmark signals are shown for the $\gamma \gamma \rightarrow \tau \tau$ samples at the SM values $\left(\delta a_{\tau}, \delta d_{\tau}\right)=(0,0)$ and for values near the threshold of $68 \%$ C.L. sensitivity $\left(\delta a_{\tau}, \delta d_{\tau}\right) \in\{(0.005,0)$, $(-0.01,0)\}$.

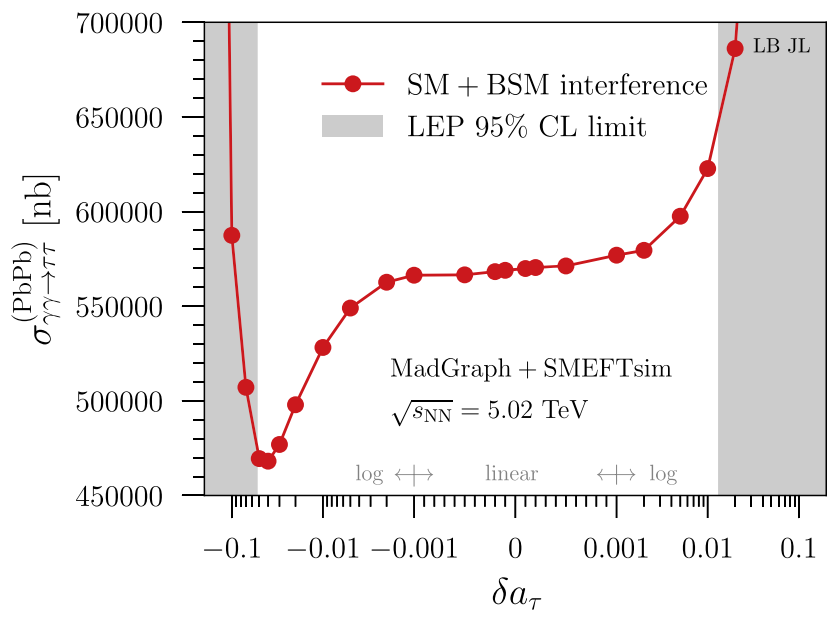

FIG. 5. Generator level cross sections for $\gamma \gamma \rightarrow \tau \tau$ sourced by our implementation of the Pb photon flux in MadGraph. This is interfaced with SMEFTsim for BSM coupling variations in $\delta a_{\tau}$ defined in Eq. (4) of the main text, fixing $\delta d_{\tau}=0$ at $\sqrt{s_{\mathrm{NN}}}=5.02 \mathrm{TeV}$. The left shows the contribution from only one BSM coupling (light blue triangles), two BSM couplings (dark blue squares), and their combined interference with the SM (red circles). The markers indicate the sampled points from $\delta a_{\tau}$. The right zooms in to the $\delta a_{\tau}$ values near zero with gray regions denoting the $95 \%$ C.L. exclusion by DELPHI, where the horizontal axis is linear scale for $\delta a_{\tau} \in[-0.001,0.001]$ and logarithmic elsewhere. 
TABLE I. Cutflow of event yields after each requirement applied sequentially, normalized to $\mathcal{L}=2 \mathrm{nb}^{-1}$ for the different analyses. For the $\gamma \gamma \rightarrow \tau \tau$ signal processes, we show these for benchmark points with parameter values labeled by $\left(\delta a_{\tau}, \delta d_{\tau}\right)$ displayed in the column header. Backgrounds are shown for various dilepton $\mu \mu, e e$ and diquark processes where the letters denote the flavor. The initial value in each cutflow is the cross section $\sigma$ times luminosity $\mathcal{L}$, followed by the efficiency $\epsilon_{\text {filter }}$ of the filter applied at generator level to the $\gamma \gamma \rightarrow \tau \tau$ samples.

\begin{tabular}{|c|c|c|c|c|c|c|c|c|c|c|}
\hline Requirement & $\tau \tau(0,0)$ & $\tau \tau(0.005,0)$ & $\tau(-0.01,0)$ & $\mu \mu$ & $e e$ & $b b$ & $c c$ & ss & ии & $d d$ \\
\hline \multicolumn{11}{|l|}{1 lepton +1 track analysis $(\mathrm{SR} 1 \ell 1 \mathrm{~T})$} \\
\hline$\sigma \times \mathcal{L}$ & 1139800 & 1195060 & 1056400 & 844080 & 844080 & 2999 & 604080 & 37754 & 604080 & 37754 \\
\hline$\sigma \times \mathcal{L} \times \epsilon_{\text {filter }}$ & 241140 & 253920 & 226300 & 844080 & 844080 & 2999 & 604080 & 37754 & 604080 & 37754 \\
\hline $1 \ell$ plus 1 track & 20492.2 & 21619.3 & 19348.4 & 263443 & 3299.3 & 5.4 & 2905.0 & 0.3 & 5.4 & 0.2 \\
\hline$p_{\mathrm{T}}^{e / \mu}>4.5 / 3 \mathrm{GeV},\left|\eta^{e / \mu}\right|<2.5 / 2.4$ & 3659.9 & 3882.7 & 3582.8 & 79043 & 3118.9 & 1.1 & 4.8 & 0.0 & 0.0 & 0.0 \\
\hline$p_{\mathrm{T}}^{\mathrm{trk}}>0.5 \mathrm{GeV},\left|\eta^{\mathrm{trk}}\right|<2.5$ & 3324.5 & 3535.9 & 3256.9 & 78973 & 3117.8 & 1.0 & 3.0 & 0.0 & 0.0 & 0.0 \\
\hline$|\Delta \phi(\ell, \operatorname{trk})|<3$ & 1519.7 & 1605.7 & 1468.3 & 0.9 & 5.3 & 0.7 & 1.8 & 0.0 & 0.0 & 0.0 \\
\hline$m_{\ell, \text { trk }} \notin\{[3,3.2],[9,11]\} \mathrm{GeV}$ & 1275.1 & 1353.6 & 1242.3 & 0.9 & 5.3 & 0.2 & 1.2 & 0.0 & 0.0 & 0.0 \\
\hline$p_{\mathrm{T}}^{\ell} \leq 6.0 \mathrm{GeV}$ & 1197.7 & 1262.3 & 1154.7 & 0.9 & 0.0 & 0.2 & 1.2 & 0.0 & 0.0 & 0.0 \\
\hline$p_{\mathrm{T}}^{\ell}>6.0 \mathrm{GeV}$ & 77.3 & 91.3 & 87.6 & 0.0 & 5.3 & 0.0 & 0.0 & 0.0 & 0.0 & 0.0 \\
\hline \multicolumn{11}{|l|}{1 lepton + multitrack analysis (SR $1 \ell 2 / 3 \mathrm{~T})$} \\
\hline$\sigma \times \mathcal{L}$ & 1139800 & 1195060 & 1056400 & 844080 & 844080 & 2999 & 604080 & 37754 & 604080 & 37754 \\
\hline$\sigma \times \mathcal{L} \times \epsilon_{\text {filter }}$ & 241140 & 253920 & 226300 & 844080 & 844080 & 2999 & 604080 & 37754 & 604080 & 37754 \\
\hline $1 \ell$ plus 2 or 3 tracks & 5945.1 & 6260.1 & 5572.2 & 33.8 & 23.2 & 43.8 & 8056.6 & 5.4 & 132.9 & 6.8 \\
\hline$p_{\mathrm{T}}^{e / \mu}>4.5 / 3 \mathrm{GeV},\left|\eta^{e / \mu}\right|<2.5 / 2.4$ & 1010.0 & 1073.3 & 978.6 & 12.2 & 4.2 & 1.8 & 13.3 & 0.0 & 0.0 & 0.0 \\
\hline$\ell+2$ tracks, $p_{\mathrm{T}}^{\mathrm{trk}}>0.5 \mathrm{GeV},|\eta|^{\mathrm{trk}}<2.5$ & 519.9 & 548.1 & 485.8 & 5.6 & 4.2 & 0.8 & 4.8 & 0.0 & 0.0 & 0.0 \\
\hline$\ell+3$ tracks, $p_{\mathrm{T}}^{\mathrm{trk}}>0.5 \mathrm{GeV},|\eta|^{\mathrm{trk}}<2.5$ & 370.5 & 398.3 & 381.1 & 0.0 & 0.0 & 0.4 & 3.6 & 0.0 & 0.0 & 0.0 \\
\hline
\end{tabular}

Figure 6 shows the $\chi^{2}$ distributions as a function of $\delta a_{\tau}$ and $\delta d_{\tau}$ assuming the other is zero for separate signal regions. These are shown assuming $10 \%$ systematics, $2 \mathrm{nb}^{-1}$ to allow comparison of constraining power between the different analyses presented in the main text.

Figure 7 displays the combined $\chi^{2}=\sum_{i} \chi_{i}^{2}$ distributions. The combined $\chi^{2}$ distributions are shown for $10 \%$ systematics at $2 \mathrm{nb}^{-1}$ together with prospects using $5 \%$ systematics and extrapolation to $20 \mathrm{nb}^{-1}$. The red lines show the results from combining the three track SRs. The final combined $\chi^{2}$ for the results in the main text take the green lines, which combine all four signal regions (SR $1 \ell 1 \mathrm{~T}$ is divided into two orthogonal $p_{\mathrm{T}}^{\ell}$ bins). The final 68\% C.L. and $95 \%$ C.L. intervals are defined by where the $\chi^{2}$ distributions intersect with $\chi^{2}=1$ and $\chi^{2}=3.84$, respectively. 

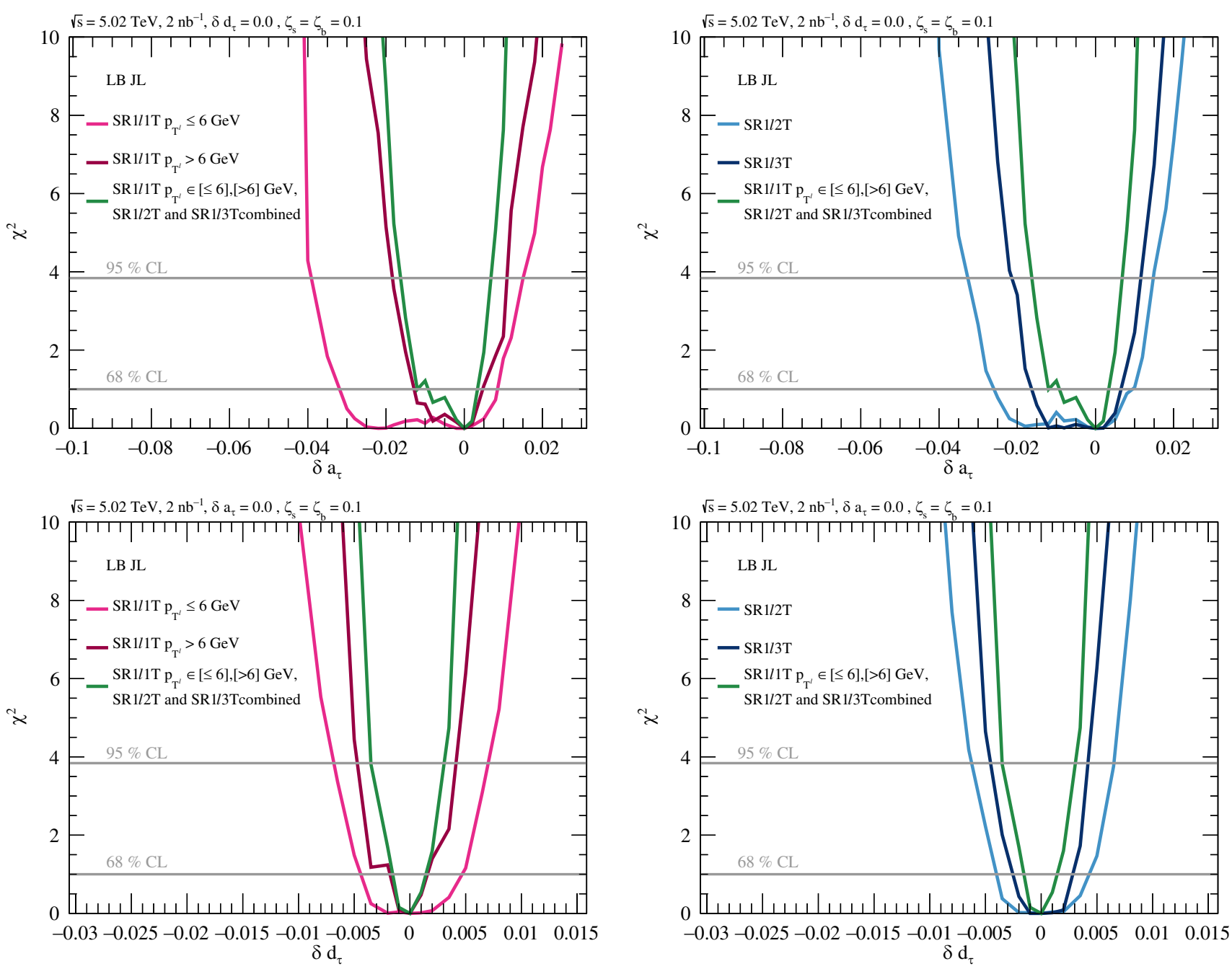

FIG. 6. The $\chi^{2}$ distributions as a function of $\delta a_{\tau}$ assuming $\delta d_{\tau}=0$ (upper) and $\delta d_{\tau}$ assuming $\delta a_{\tau}=0$ (lower) are displayed for 10\% systematics at $\mathcal{L}=2 \mathrm{nb}^{-1}$. The left shows the results from the SR $\ell \ell 1 \mathrm{~T}$ regions and the impact of binning in $p_{\mathrm{T}}^{\ell}$. The right shows the results from the SR $1 \ell 2 / 3 \mathrm{~T}$ regions. The $\chi^{2}$ combining the four signal regions is shown by the green line for reference. The gray horizontal lines correspond to 68\% C.L. $\left(\chi^{2}=1\right)$ and 95\% C.L. $\left(\chi^{2}=3.84\right)$. The unphysical spikes are due to limited Monte Carlo statistics. 

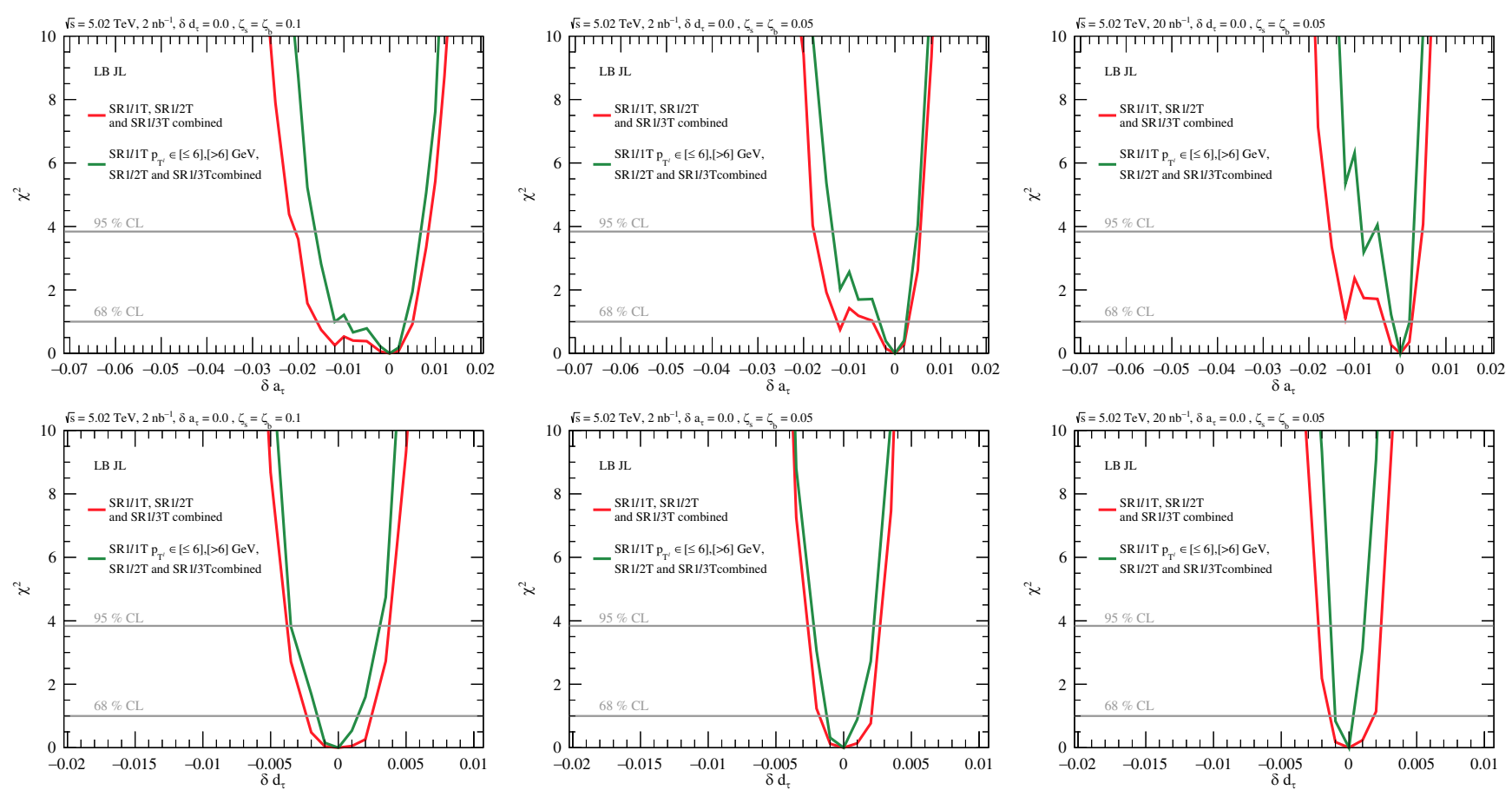

FIG. 7. The $\chi^{2}$ distributions as a function of $\delta a_{\tau}$ assuming $\delta d_{\tau}=0$ (upper) and $\delta d_{\tau}$ assuming $\delta a_{\tau}=0$ (lower). These are displayed for $10 \%$ (left), $5 \%$ (center) systematics at $\mathcal{L}=2 \mathrm{nb}^{-1}$, and $5 \%$ systematics result extrapolated to $\mathcal{L}=20 \mathrm{nb}^{-1}$ (right). The combined $\chi^{2}$ for all three track SRs is shown by the red line, while the impact of dividing SR1 $\ell$ 1T into two orthogonal $p_{\mathrm{T}}^{\ell}$ bins is shown by the green line. The gray horizontal lines correspond to $68 \%$ C.L. $\left(\chi^{2}=1\right)$ and 95\% C.L. $\left(\chi^{2}=3.84\right)$. The unphysical spikes are due to limited Monte Carlo statistics.

[1] B. Odom, D. Hanneke, B. D'Urso, and G. Gabrielse, New Measurement of the Electron Magnetic Moment Using a One-Electron Quantum Cyclotron, Phys. Rev. Lett. 97, 030801 (2006).

[2] D. Hanneke, S. F. Hoogerheide, and G. Gabrielse, Cavity control of a single-electron quantum cyclotron: Measuring the electron magnetic moment, Phys. Rev. A 83, 052122 (2011).

[3] R. Bouchendira, P. Cladé, S. Guellati-Khélifa, F. Nez, and F. Biraben, New Determination of the Fine Structure Constant and Test of the Quantum Electrodynamics, Phys. Rev. Lett. 106, 080801 (2011).

[4] T. Aoyama, M. Hayakawa, T. Kinoshita, and M. Nio, Tenth-Order QED Contribution to the Electron g-2 and an Improved Value of the Fine Structure Constant, Phys. Rev. Lett. 109, 111807 (2012).

[5] R. H. Parker, C. Yu, W. Zhong, B. Estey, and H. Müller, Measurement of the fine-structure constant as a test of the Standard Model, Science 360, 191 (2018).

[6] G. W. Bennett et al. (Muon g-2 Collaboration), Final report of the Muon E821 anomalous magnetic moment measurement at BNL, Phys. Rev. D 73, 072003 (2006).
[7] T. Aoyama, M. Hayakawa, T. Kinoshita, and M. Nio, Complete Tenth-Order QED Contribution to the Muon g-2, Phys. Rev. Lett. 109, 111808 (2012).

[8] A. Keshavarzi, D. Nomura, and T. Teubner, Muon $g-2$ and $\alpha\left(M_{Z}^{2}\right)$ : A new data-based analysis, Phys. Rev. D 97, 114025 (2018).

[9] M. Davier, A. Hoecker, B. Malaescu, and Z. Zhang, A new evaluation of the hadronic vacuum polarisation contributions to the muon anomalous magnetic moment and to $\boldsymbol{\alpha}\left(\mathbf{m}_{\mathbf{Z}}^{\mathbf{2}}\right)$, Eur. Phys. J. C 80, 241 (2020).

[10] S. P. Martin and J. D. Wells, Muon anomalous magnetic dipole moment in supersymmetric theories, Phys. Rev. D 64, 035003 (2001).

[11] A. Czarnecki and W. J. Marciano, The Muon anomalous magnetic moment: A Harbinger for 'new physics', Phys. Rev. D 64, 013014 (2001).

[12] K. Hagiwara, R. Liao, A. D. Martin, D. Nomura, and T. Teubner, $(g-2)_{\mu}$ and $\alpha\left(M_{Z}^{2}\right)$ re-evaluated using new precise data, J. Phys. G 38, 085003 (2011).

[13] M. A. Ajaib, B. Dutta, T. Ghosh, I. Gogoladze, and Q. Shafi, Neutralinos and sleptons at the LHC in light of muon $(g-2)_{\mu}$, Phys. Rev. D 92, 075033 (2015). 
[14] J. Grange et al. (Muon g-2 Collaboration), Muon (g-2) technical design report, arXiv:1501.06858.

[15] M. Abe et al., A new approach for measuring the muon anomalous magnetic moment and electric dipole moment, Prog. Theor. Exp. Phys. 053C02 (2019) .

[16] D. J. Silverman and G. L. Shaw, Limits on the composite structure of the tau lepton and quarks from anomalous magnetic moment measurements in $e^{+} e^{-}$annihilation, Phys. Rev. D 27, 1196 (1983).

[17] J. Abdallah et al. (DELPHI Collaboration), Study of taupair production in photon-photon collisions at LEP and limits on the anomalous electromagnetic moments of the tau lepton, Eur. Phys. J. C 35, 159 (2004).

[18] M. Tanabashi et al. (Particle Data Group), Review of particle physics, Phys. Rev. D 98, 030001 (2018).

[19] S. Eidelman and M. Passera, Theory of the tau lepton anomalous magnetic moment, Mod. Phys. Lett. A 22, 159 (2007).

[20] B. Dutta and Y. Mimura, Electron $g-2$ with flavor violation in MSSM, Phys. Lett. B 790, 563 (2019).

[21] H. Davoudiasl and W. J. Marciano, Tale of two anomalies, Phys. Rev. D 98, 075011 (2018).

[22] M. Bauer, M. Neubert, S. Renner, M. Schnubel, and A. Thamm, Axion-Like Particles, Lepton-Flavor Violation and a New Explanation of $a_{\mu}$ and $a_{e}$, Phys. Rev. Lett. 124, 211803 (2020).

[23] M. Endo and W. Yin, Explaining electron and muon $g-2$ anomaly in SUSY without lepton-flavor mixings, J. High Energy Phys. 08 (2019) 122.

[24] LHCb Collaboration, Measurement of the Ratio of Branching Fractions $\mathcal{B}\left(\bar{B}^{0} \rightarrow D^{*+} \tau^{-} \bar{\nu}_{\tau}\right) / \mathcal{B}\left(\bar{B}^{0} \rightarrow D^{*+} \mu^{-} \bar{\nu}_{\mu}\right)$, Phys. Rev. Lett. 115, 111803 (2015); Erratum, Phys. Rev. Lett. 115, 159901 (2015).

[25] Belle Collaboration, Measurement of $\mathcal{R}(D)$ and $\mathcal{R}\left(D^{*}\right)$ with a semileptonic tagging method, arXiv:1904.08794.

[26] B. Allanach, F. S. Queiroz, A. Strumia, and S. Sun, $Z^{\prime}$ models for the $\mathrm{LHCb}$ and $g-2$ muon anomalies, Phys. Rev. D 93, 055045 (2016); Erratum, Phys. Rev. D 95, 119902 (2017).

[27] S. Di Chiara, A. Fowlie, S. Fraser, C. Marzo, L. Marzola, M. Raidal, and C. Spethmann, Minimal flavor-changing $Z^{\prime}$ models and muon $g-2$ after the $R_{K^{*}}$ measurement, Nucl. Phys. B923, 245 (2017).

[28] A. Biswas and A. Shaw, Reconciling dark matter, $R_{K^{(*)}}$ anomalies and $(g-2)_{\mu}$ in an $L_{\mu}-L_{\tau}$ scenario, J. High Energy Phys. 05 (2019) 165.

[29] W. Yin and N. Yokozaki, Splitting mass spectra and muon g-2 in Higgs-anomaly mediation, Phys. Lett. B 762, 72 (2016).

[30] M. Yamaguchi and W. Yin, A novel approach to finely tuned supersymmetric standard models: The case of the non-universal Higgs mass model, Prog. Theor. Exp. Phys. 023B06 (2018).

[31] T. Fukuyama, A. Ilakovac, and T. Kikuchi, Lepton flavor violating leptonic/semileptonic decays of charged leptons in the minimal supersymmetric standard model, Eur. Phys. J. C 56, 125 (2008).

[32] K. S. Babu, T. Fukuyama, S. Khan, and S. Saad, PecceiQuinn symmetry and nucleon decay in renormalizable SUSY SO(10), J. High Energy Phys. 06 (2019) 045.
[33] F. del Aguila, F. Cornet, and J. I. Illana, The possibility of using a large heavy-ion collider for measuring the electromagnetic properties of the tau lepton, Phys. Lett. B 271, 256 (1991).

[34] M. Koksal, A. A. Billur, A. Gutiérrez-Rodríguez, and M. A. Hernández-Ruíz, Model-independent sensitivity estimates for the electromagnetic dipole moments of the $\tau$-lepton at the CLIC, Phys. Rev. D 98, 015017 (2018).

[35] A. A. Billur and M. Köksal, Probe of the electromagnetic moments of the tau lepton in gamma-gamma collisions at the CLIC, Phys. Rev. D 89, 037301 (2014).

[36] J. N. Howard, A. Rajaraman, R. Riley, and T. M. P. Tait, The $\tau$ magnetic dipole moment at future lepton colliders, Lett. High Energy Phys. 2, 5 (2019).

[37] M. Koksal, Search for the electromagnetic moments of the $\tau$ lepton in photon-photon collisions at the $\mathrm{LHeC}$ and the FCC-he, J. Phys. G 46, 065003 (2019).

[38] A. Gutiérrez-Rodríguez, M. Köksal, A. A. Billur, and M. A. Hernández-Ruíz, Feasibility at the LHC, FCC-he and CLIC for sensitivity estimates on anomalous $\tau$-lepton couplings, arXiv:1903.04135.

[39] M. Fael, L. Mercolli, and M. Passera, Towards a determination of the tau lepton dipole moments, Nucl. Phys. B, Proc. Suppl. 253-255, 103 (2014).

[40] S. Eidelman, D. Epifanov, M. Fael, L. Mercolli, and M. Passera, $\tau$ dipole moments via radiative leptonic $\tau$ decays, J. High Energy Phys. 03 (2016) 140.

[41] X. Chen and Y. Wu, Search for the Electric Dipole Moment and anomalous magnetic moment of the tau lepton at tau factories, J. High Energy Phys. 10 (2019) 089.

[42] M. A. Samuel and G. Li, How to measure the magnetic moment of the tau lepton, Int. J. Theor. Phys. 33, 1471 (1994).

[43] A. Hayreter and G. Valencia, Constraining $\tau$-lepton dipole moments and gluon couplings at the LHC, Phys. Rev. D 88, 013015 (2013); Erratum, Phys. Rev. D 91, 099902 (2015).

[44] S. Atag and A. A. Billur, Possibility of determining $\tau$ lepton electromagnetic moments in $\gamma \gamma \rightarrow \tau^{+} \tau^{-}$process at the CERN-LHC, J. High Energy Phys. 11 (2010) 060.

[45] I. Galon, A. Rajaraman, and T. M. P. Tait, $H \rightarrow \tau^{+} \tau^{-} \gamma$ as a probe of the $\tau$ magnetic dipole moment, J. High Energy Phys. 12 (2016) 111.

[46] A. S. Fomin, A. Yu Korchin, A. Stocchi, S. Barsuk, and P. Robbe, Feasibility of $\tau$-lepton electromagnetic dipole moments measurement using bent crystal at the LHC, J. High Energy Phys. 03 (2019) 156.

[47] J. Fu, M. A. Giorgi, L. Henry, D. Marangotto, F. M. Vidal, A. Merli, N. Neri, and J. R. Vidal, Novel Method for the Direct Measurement of the $\tau$ Lepton Dipole Moments, Phys. Rev. Lett. 123, 011801 (2019).

[48] ATLAS Collaboration, Back-to-back electron-muon pair in an ultra-peripheral collision recorded with the ATLAS detector, Technical Report No. ATLAS-EVENTDISPLAY2018-009, 2018.

[49] ATLAS Collaboration, Search for electroweak production of supersymmetric states in scenarios with compressed mass spectra at $\sqrt{s}=13 \mathrm{TeV}$ with the ATLAS detector, Phys. Rev. D 97, 052010 (2018).

[50] CMS Collaboration, Search for new physics in events with two soft oppositely charged leptons and missing transverse 
momentum in proton-proton collisions at $\sqrt{s}=13 \mathrm{TeV}$, Phys. Lett. B 782, 440 (2018).

[51] ATLAS Collaboration, Searches for electroweak production of supersymmetric particles with compressed mass spectra in $\sqrt{s}=13 \mathrm{TeV} p p$ collisions with the ATLAS detector, Technical Report No. ATLAS-CONF-2019-014, 2019.

[52] K. Piotrzkowski, Tagging two photon production at the CERN LHC, Phys. Rev. D 63, 071502(R) (2001).

[53] M. G. Albrow et al. (FP420 R \& D Collaboration), The FP420 R \& D Project: Higgs and New Physics with forward protons at the LHC, J. Instrum. 4, T10001 (2009).

[54] J. de Favereau de Jeneret, V. Lemaitre, Y. Liu, S. Ovyn, T. Pierzchala, K. Piotrzkowski, X. Rouby, N. Schul, and M. V. Donckt, High energy photon interactions at the LHC, arXiv:0908.2020.

[55] D. d'Enterria and G. G. da Silveira, Observing Light-byLight Scattering at the Large Hadron Collider, Phys. Rev. Lett. 111, 080405 (2013); Erratum, Phys. Rev. Lett. 116, 129901 (2016).

[56] ATLAS Collaboration, Evidence for light-by-light scattering in heavy-ion collisions with the ATLAS detector at the LHC, Nat. Phys. 13, 852 (2017).

[57] CMS Collaboration, Evidence for light-by-light scattering and searches for axion-like particles in ultraperipheral $\mathrm{PbPb}$ collisions at $\sqrt{s_{\mathrm{NN}}}=5.02 \mathrm{TeV}$, Phys. Lett. B 797, 134826 (2019).

[58] ATLAS Collaboration, Observation of Light-by-Light Scattering in Ultraperipheral $\mathrm{Pb}+\mathrm{Pb}$ Collisions with the ATLAS Detector, Phys. Rev. Lett. 123, 052001 (2019).

[59] ATLAS Collaboration, Measurement of the exclusive $\gamma \gamma \rightarrow$ $\mu^{+} \mu^{-}$process in proton-proton collisions at $\sqrt{s}=13 \mathrm{TeV}$ with the ATLAS detector, Phys. Lett. B 777, 303 (2018).

[60] ATLAS Collaboration, Measurement of exclusive $\gamma \gamma \rightarrow$ $W^{+} W^{-}$production and search for exclusive Higgs boson production in $p p$ collisions at $\sqrt{s}=8 \mathrm{TeV}$ using the ATLAS detector, Phys. Rev. D 94, 032011 (2016).

[61] CMS Collaboration, Search for exclusive or semiexclusive photon pair production and observation of exclusive and semi-exclusive electron pair production in $p p$ collisions at $\sqrt{s}=7 \mathrm{TeV}$, J. High Energy Phys. 11 (2012) 080.

[62] CMS Collaboration, Evidence for exclusive $\gamma \gamma \rightarrow W^{+} W^{-}$ production and constraints on anomalous quartic gauge couplings in $p p$ collisions at $\sqrt{s}=7$ and $8 \mathrm{TeV}$, J. High Energy Phys. 08 (2016) 119.

[63] ATLAS Collaboration, Measurement of high-mass dimuon pairs from ultraperipheral lead-lead collisions at $\sqrt{s_{\mathrm{NN}}}=$ $5.02 \mathrm{TeV}$ with the ATLAS detector, Technical Report No. ATLAS-CONF-2016-025, 2016.

[64] E. Chapon, C. Royon, and O. Kepka, Anomalous quartic $W W \gamma \gamma, Z Z \gamma \gamma$, and trilinear $W W \gamma$ couplings in two-photon processes at high luminosity at the LHC, Phys. Rev. D 81, 074003 (2010).

[65] S. Fichet, G. von Gersdorff, O. Kepka, B. Lenzi, C. Royon, and M. Saimpert, Probing new physics in diphoton production with proton tagging at the Large Hadron Collider, Phys. Rev. D 89, 114004 (2014).

[66] J. Ellis, N. E. Mavromatos, and T. You, Light-by-Light Scattering Constraint on Born-Infeld Theory, Phys. Rev. Lett. 118, 261802 (2017).
[67] S. Knapen, T. Lin, H. K. Lou, and T. Melia, Searching for Axionlike Particles with Ultraperipheral Heavy-Ion Collisions, Phys. Rev. Lett. 118, 171801 (2017).

[68] C. Baldenegro, S. Fichet, G. von Gersdorff, and C. Royon, Searching for axion-like particles with proton tagging at the LHC, J. High Energy Phys. 06 (2018) 131.

[69] J. Ohnemus, T. F. Walsh, and P. M. Zerwas, gamma gamma production of nonstrongly interacting SUSY particles at hadron colliders, Phys. Lett. B 328, 369 (1994).

[70] N. Schul and K. Piotrzkowski, Detection of two-photon exclusive production of supersymmetric pairs at the LHC, Nucl. Phys. B, Proc. Suppl. 179-180, 289 (2008).

[71] L. A. Harland-Lang, C. H. Kom, K. Sakurai, and W. J. Stirling, Measuring the masses of a pair of semi-invisibly decaying particles in central exclusive production with forward proton tagging, Eur. Phys. J. C 72, 1969 (2012).

[72] L. Beresford and J. Liu, Photon Collider Search Strategy for Sleptons and Dark Matter at the LHC, Phys. Rev. Lett. 123, 141801 (2019).

[73] L. A. Harland-Lang, V. A. Khoze, M. G. Ryskin, and M. Tasevsky, LHC searches for dark matter in compressed mass scenarios: Challenges in the forward proton mode, J. High Energy Phys. 04 (2019) 010.

[74] R. Bruce et al., New physics searches with heavy-ion collisions at the LHC, J. Phys. G 47, 060501 (2020).

[75] R. Escribano and E. Masso, New bounds on the magnetic and electric moments of the tau lepton, Phys. Lett. B 301, 419 (1993).

[76] B. Grzadkowski, M. Iskrzynski, M. Misiak, and J. Rosiek, Dimension-six terms in the standard model lagrangian, J. High Energy Phys. 10 (2010) 085.

[77] S. J. Brodsky, T. Kinoshita, and H. Terazawa, Two photon mechanism of particle production by high-energy colliding beams, Phys. Rev. D 4, 1532 (1971).

[78] G. Tupper and M. A. Samuel, $W^{+} W^{-}$pair production in two-photon collisions and the magnetic moment of the $W^{ \pm}$ bosons, Phys. Rev. D 23, 1933 (1981).

[79] I. Brivio, Y. Jiang, and M. Trott, The SMEFTsim package, theory and tools, J. High Energy Phys. 12 (2017) 070.

[80] A. Alloul, N. D. Christensen, C. Degrande, C. Duhr, and B. Fuks, FeynRules 2.0-A complete toolbox for tree-level phenomenology, Comput. Phys. Commun. 185, 2250 (2014).

[81] J. Alwall, M. Herquet, F. Maltoni, O. Mattelaer, and T. Stelzer, MadGraph 5: Going beyond, J. High Energy Phys. 06 (2011) 128.

[82] J. Alwall, R. Frederix, S. Frixione, V. Hirschi, F. Maltoni, O. Mattelaer, H.-S. Shao, T. Stelzer, P. Torrielli, and M. Zaro, The automated computation of tree-level and nextto-leading order differential cross sections, and their matching to parton shower simulations, J. High Energy Phys. 07 (2014) 079.

[83] V. M. Budnev, I. F. Ginzburg, G. V. Meledin, and V. G. Serbo, The Two photon particle production mechanism. Physical problems. Applications. Equivalent photon approximation, Phys. Rep. 15, 181 (1975).

[84] D. d'Enterria and J.-P. Lansberg, Study of Higgs boson production and its $\mathrm{b}$ anti-b decay in gamma-gamma processes in proton-nucleus collisions at the LHC, Phys. Rev. D 81, 014004 (2010). 
[85] J. D. Jackson, Classical Electrodynamics; 3rd ed. (Wiley, New York, NY, 1999).

[86] S. Zhang and J. M. Jin, Computation of Special Functions, A Wiley-Interscience publication (Wiley, New York, 1996).

[87] L. A. Harland-Lang, V. A. Khoze, and M. G. Ryskin, Exclusive LHC physics with heavy ions: SuperChic 3, Eur. Phys. J. C 79, 39 (2019).

[88] T. Sjostrand, S. Mrenna, and P.Z. Skands, A brief introduction to PYTHIA 8.1, Comput. Phys. Commun. 178, 852 (2008).

[89] J. de Favereau, C. Delaere, P. Demin, A. Giammanco, V. Lemaître, A. Mertens, and M. Selvaggi (DELPHES 3 Collaboration), DELPHES 3, A modular framework for fast simulation of a generic collider experiment, J. High Energy Phys. 02 (2014) 057.

[90] ATLAS Collaboration, Performance of the ATLAS trigger system in 2015, Eur. Phys. J. C 77, 317 (2017).

[91] ATLAS Collaboration, The ATLAS tau trigger in run 2, Technical Report No. ATLAS-CONF-2017-061, 2017.

[92] ATLAS Collaboration, Performance of the ATLAS minimum bias and forward detector triggers in 2011 heavy ion run, Technical Report No. ATLAS-CONF-2012-122, 2012.

[93] ATLAS Collaboration, Performance of the ATLAS minimum bias and forward detector triggers in $\mathrm{pPb}$ collisions, Technical Report No. ATLAS-CONF-2013104, 2013.
[94] ATLAS Collaboration, Zero degree calorimeters for ATLAS, Technical Report No. CERN-LHCC-2007-01, 2007.

[95] CMS Collaboration, Observation of forward neutron multiplicity dependence of dimuon acoplanarity in ultraperipheral $\mathrm{PbPb}$ collisions at $\sqrt{s_{\mathrm{NN}}}=5.02 \mathrm{TeV}$, arXiv:2011.05239.

[96] ATLAS Collaboration, Electron reconstruction and identification in the ATLAS experiment using the 2015 and 2016 LHC proton-proton collision data at $\sqrt{s}=13 \mathrm{TeV}$, Eur. Phys. J. C 79, 639 (2019).

[97] ATLAS Collaboration, Muon reconstruction performance of the ATLAS detector in proton-proton collision data at $\sqrt{s}=13 \mathrm{TeV}$, Eur. Phys. J. C 76, 292 (2016).

[98] M. E. Pospelov and I. B. Khriplovich, Electric dipole moment of the $\mathrm{W}$ boson and the electron in the KobayashiMaskawa model, Yad. Fiz. 53, 1030 (1991) [Sov. J. Nucl. Phys. 53, 638 (1991)].

[99] D. Ng and J. N. Ng, A note on majorana neutrinos, leptonic CKM and electron electric dipole moment, Mod. Phys. Lett. A 11, 211 (1996).

[100] K. Inami et al. (Belle Collaboration), Search for the electric dipole moment of the tau lepton, Phys. Lett. B 551, 16 (2003).

[101] CMS Collaboration, Technical proposal for MIP timing detector in the CMS experiment phase 2 upgrade, Technical Report No. CERN-LHCC-2017-027, 2017.

[102] W. Yu (ALICE Collaboration), Particle identification of the ALICE TPC via dE/dx, Nucl. Instrum. Methods Phys. Res., Sect. A 706, 55 (2013). 